\title{
Stability of Biorelevant Media Under Various Storage Conditions
}

\author{
Lukas Klumpp ${ }^{1}$, Kalpa Nagasekar ${ }^{1}$, Orla McCullough², Anja Seybert ${ }^{3}$, Mukul Ashtikar ${ }^{4}$, and Jennifer \\ Dressman ${ }^{*}$ \\ ${ }^{1}$ Institute of Pharmaceutical Technology, Goethe University, Frankfurt am Main, Germany \\ ${ }^{2}$ Biorelevant.com Ltd, 41 New Road, London, UK \\ ${ }^{3}$ Buchmann Institute for Molecular Life Sciences, Goethe University, Frankfurt am Main, Germany \\ ${ }^{4}$ Fraunhofer Institute for Molecular Biology and Applied Ecology, Branch Translational Medicinal Pharmacology, Frankfurt am Main, Germany
}

e-mail:dressman@em.uni-frankfurt.de

\begin{abstract}
The physical and chemical stability of various biorelevant media (FaSSGF, FaSSIF V1, FaSSIF V2, FaSSIF V3, and FeSSIF) were investigated over periods after preparation of up to $120 \mathrm{~h}$ at room temperature (RT) $\left(22^{\circ} \mathrm{C}\right)$ and $37^{\circ} \mathrm{C}$. It was shown that biorelevant instant powders are not a source of microbiological contamination. It was also established that the physical characteristics of FaSSGF and FeSSIF are invariant with time, so they can be used immediately after preparation, i.e., without any equilibration time. Alternatively, they can be stored for up to $96 \mathrm{~h}$ at RT before use. By contrast, FaSSIF V1 requires an equilibration time of $2 \mathrm{~h}$, and FaSSIF V2 requires an equilibration time of $24 \mathrm{~h}$ to enable the media characteristics to stabilize. After this equilibration time, both FaSSIF V1 and V2 can also be stored for at least up to $96 \mathrm{~h}$ at RT prior to use; longer storage times have not yet been tested. The particle size of FaSSIF V3 appears to continually evolve over time. For FaSSIF V3, it is thus recommended that the waiting period between preparation and use be standardized among experiments. Additionally, the colloidal particle structures present in the various FaSSIF versions were analyzed by cryogenic transmission electron microscopy (Cryo-TEM). Various kinds of micelles (globular, disc, multilayer disc, and thread-like micelles) were observed in all samples, and some samples also contained unilamellar vesicles. Differences in solubility of drugs among the various FaSSIF versions can be partly explained by the results from Cryo-TEM.
\end{abstract}

KEYWORDS: Biorelevant media, stability, Cryo-TEM, microbiological, particle size, dissolution

\section{INTRODUCTION}

B iorelevant media are a common tool in drug development and quality control in research labs and the pharmaceutical industry. Since the first publication in 1998, biorelevant media have been updated according to the latest knowledge of the gastrointestinal (GI) tract physiology, leading to an array of media versions that are available to simulate conditions in various segments of the GI tract (1). Biorelevant media include fasted-state gastric media (FaSSGF), three versions of fasted-state intestinal media (FaSSIF V1, FaSSIF V2, FaSSIF V3), and fed-state intestinal media (FeSSIF). Factors such as the drug solubility under GI conditions, release from the dosage form, the potential for precipitation subsequent to release, food effects, stability of the drug upon exposure to Gl fluids, and permeability through the intestinal membrane can all be tested under biorelevant conditions with these media (2). The knowledge gained from these experiments leads to a better understanding of the drug's rate and extent of absorption, thus streamlining development and reducing costs (3). Compared to compendial media, biorelevant media mimic the physiological conditions more accurately for many drug-drug product combinations (4).

When biorelevant media were first introduced, it was necessary for the user to prepare the media freshly from the individual ingredients for each experiment. In the meantime, instant powder versions of several biorelevant media have become commercially available, obviating the need to manufacture them onsite, and they are now as simple to prepare as compendial media.

The question has been raised as to whether these commercially available versions of biorelevant media can generate reproducible and reliable results among batches, or if small differences in the composition, which

* Corresponding author. 
could occur during manufacturing, would have a negative influence on reproducibility. In a recent ring study, 16 industrial and academic laboratories utilized a total of 11 different batches of the commercially available instant powder that can be used to produce FaSSGF, FaSSIF V1, and FeSSIF (FaSSIF/FeSSIF/FaSSGF instant powder) in dissolution and two-stage release experiments (5). Almost all results were within a 95\% confidence interval, with just a few outliers that could be explained by lack of adherence to the experimental protocol. The authors concluded that, in a practical laboratory setting, biorelevant media can be used to obtain reproducible and reliable results. However, in those experiments the media were prepared on the day of the experiment, and it has not yet been clarified whether this protocol must be followed for the instant powders or if they can be stored for several days after reconstitution and still maintain their physicochemical properties and release behavior.

In this work, we examined certain key properties of biorelevant media upon reconstitution as well as under conditions of storage and use. The aim was to increase our understanding of the dynamic behavior of these media and to establish the minimum shelf life of the reconstituted media under typical conditions of storage and use. Specifically, the physical and chemical properties of FaSSGF, FaSSIF V1, FaSSIF V2, FaSSIF V3, and FeSSIF were investigated over periods of up to $96 \mathrm{~h}$ at room temperature (RT) $\left(22^{\circ} \mathrm{C}\right)$ with an additional $24 \mathrm{~h}$ at the end of the storage period under conditions applied in solubility studies, i.e., $37^{\circ} \mathrm{C}$. To gain further understanding of their behavior on a microscopic scale, the three different FaSSIF versions were also studied using cryogenic transmission electron microscopy (Cryo-TEM).

\section{MATERIALS AND METHODS}

All chemicals used were of analytical grade. The instant powders, FaSSIF/FeSSIF/FaSSGF-Powder (six batches), FaSSIF V2 (one batch), and FaSSIF V3 (one batch) were kindly donated by Biorelevant.com Ltd. (London, United Kingdom). For microbiological stability studies, ready-touse sterile tryptone soya broth medium was purchased from VWR Chemicals (Darmstadt, Germany). For electron microscopic analysis, copper grids with Lacey-Carbonfilm were purchased from Plano GmbH (Wetzlar, Germany). All commercially available media were prepared according to the standard operating procedures provided by Biorelevant.com Ltd. except for FaSSIF V3, which was prepared as described by Fuchs et al. $(6,7)$. Table 1 shows an overview of the media composition.
Table 1. Composition of the Different Biorelevant Media.

\begin{tabular}{|l|c|c|c|c|c|}
\hline & FaSSGF & $\begin{array}{c}\text { FaSSIF } \\
\text { V1 }\end{array}$ & $\begin{array}{c}\text { FaSSIF } \\
\text { V2 }\end{array}$ & $\begin{array}{c}\text { FaSSIF } \\
\text { V3 }\end{array}$ & FeSSIF \\
\hline Cholesterol (mM) & - & - & - & 0.2 & - \\
\hline Lecithin (mM) & 0.02 & 0.75 & 0.2 & 0.035 & 3.75 \\
\hline Lysolecithin (mM) & - & - & - & 0.315 & - \\
\hline $\begin{array}{l}\text { Sodium } \\
\text { glycocholate (mM) }\end{array}$ & - & - & - & 1.4 & - \\
\hline Sodium oleat (mM) & - & - & - & 0.315 & - \\
\hline $\begin{array}{l}\text { Sodium } \\
\text { taurocholate (mM) }\end{array}$ & 0.08 & 3 & 3 & 1.4 & 15 \\
\hline Acetic acid (mM) & - & - & - & - & 144 \\
\hline Hydrochloric acid & $\mathrm{qs} \mathrm{pH} \mathrm{1.6}$ & - & - & - & - \\
\hline Maleic acid (mM) & - & - & 19.12 & 10.26 & - \\
\hline $\begin{array}{l}\text { Potassium } \\
\text { dihydrogen } \\
\text { phosphate (mM) }\end{array}$ & - & 28.65 & - & - & - \\
\hline $\begin{array}{l}\text { Sodium chloride } \\
\text { (mM) }\end{array}$ & 34.2 & 105.85 & 68.62 & 93.3 & 203.18 \\
\hline $\begin{array}{l}\text { Sodium hydroxide } \\
\text { (mM) }\end{array}$ & - & 10.5 & 34.8 & 16.56 & 101 \\
\hline
\end{tabular}

FaSSGF, fasted-state gastric media; FaSSIF V1-V3, fasted-state intestinal media version 1-3; FeSSIF, fed-state intestinal media; qs, quantum satis (as much as required).

\section{Stability and Quality Tests}

To evaluate the quality and stability of the biorelevant media, freshly prepared media were subjected to an initial assessment and assessment during and after several days of storage at two storage temperatures ( $n=3$ for each time/storage condition), as shown in Figure 1.

The parameters that were studied to evaluate the quality of the biorelevant media after various storage times included $\mathrm{pH}$, buffer capacity, surface tension, turbidity, particle size, and particle size distribution.

Initial testing: According to the instructions provided by Biorelevant.com Ltd., all reconstituted media must be maintained at RT for $2 \mathrm{~h}$ after preparation before use. Therefore, for the initial assessment, the media were first stored for $2 \mathrm{~h}$ at RT $\left(22^{\circ} \mathrm{C}\right)$. Differences in the various parameters from just after reconstitution (i.e. the initial value) to the end of the waiting period ( $2 \mathrm{~h}$ after preparation) were studied to determine the need for an equilibration period. After $2 \mathrm{~h}$, the media were then incubated at $37{ }^{\circ} \mathrm{C}$ for a further $22 \mathrm{~h}$ in a B12 microbiological incubator (Heraeus, Hanau, Germany). Although testing with biorelevant media representing the fasted state small intestine at $37^{\circ} \mathrm{C}$ usually occurs over a period of $30 \mathrm{~min}$ to $4 \mathrm{~h}$, these conditions were chosen to 
a) Initial Assessment

b) Assessment During And After Extended Storage At Room Temperature

c) Cryo-TEM Assessment i)

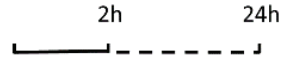

$6 \min 2 \mathrm{~h} \quad 24 \mathrm{~h} \quad 48 \mathrm{~h} \quad 96 \mathrm{~h} \quad 120 \mathrm{~h}$

ii)

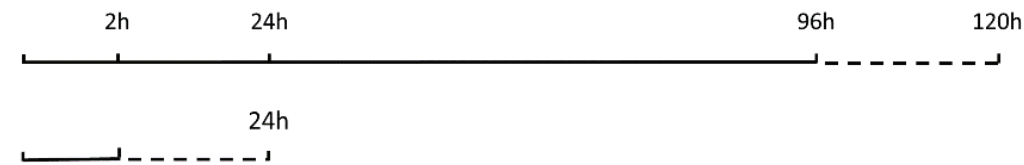

Room Temperature (approx. $22^{\circ} \mathrm{C}$ )

$-----37^{\circ} \mathrm{C}$

Figure 1. Sampling points of the different assessments. Solid line represents storage at room temperature (approximately $22{ }^{\circ} \mathrm{C}$ ), and the dotted line indicates storage at $37^{\circ} \mathrm{C}$. Times at which samples were taken are shown on the timeline. Cryo-TEM, cryogenic transmission electronic microscopy.

correspond to the maximum duration of release testing of oral dosage forms as well as usual incubation times for solubility experiments.

Extended storage: To explore the possibility that the media could be reconstituted and then held at RT for several days before use in release testing, the media was stored at RT over a period of $96 \mathrm{~h}$ and sampled after 24 , 48 , and $96 \mathrm{~h}$. Physicochemical properties after $96 \mathrm{~h}$ of storage were compared with the properties after the 2 $\mathrm{h}$ equilibration time. This part of the study established how long the media can be kept at RT before use, without compromising their quality. After the media had been stored for $96 \mathrm{~h}$ at RT, they were incubated at $37^{\circ} \mathrm{C}$ for a further $24 \mathrm{~h}$ in a B12 incubator and the parameters were assessed again. A comparison was also made between samples that had been exposed to $37^{\circ} \mathrm{C}$ for $24 \mathrm{~h}$ after storing at RT for either 2 or $96 \mathrm{~h}$.

To identify any changes at the microscopic level during equilibration, storage and release testing, samples of the three versions of FaSSIF were taken at various time and temperature combinations and evaluated by Cryo-TEM.

\section{Buffer Capacity}

The buffer capacity of FaSSIF V1, FaSSIF V2, FaSSIF V3, and FeSSIF was measured in triplicate by titration with $1 \mathrm{M}$ hydrochloric acid $(\mathrm{HCl})(8)$. Measured values were compared to previously published data (9).

\section{Surface Tension}

The surface tension of the media was measured with a Delta-8 multichannel microtensiometer $(n=8)$ and an AquaPi portable tensiometer $(n=3)$ by Kibron Inc. (Helsinki, Finland). Measurements were carried out at $19{ }^{\circ} \mathrm{C}(7,9)$.

\section{Turbidity}

Turbidity was measured with an Orion AQ3010 turbidity instrument from Thermo Scientific (Dratford, United Kingdom). The instrument was calibrated on each day of use with a styrene divinylbenzene primary standard provided by Thermo Scientific. Results are reported in nephelometric turbidity units (NTU).

\section{Particle Size and Polydispersity Index}

Particle size distributions were measured using a Zetasizer Nano ZS by Malvern Instruments (Worcestershire, United Kingdom) via photon correlation spectroscopy (PCS). All samples were analyzed without dilution.

\section{Microbiological Analysis}

The aim of these experiments was to determine whether the instant powder versions of the biorelevant media are a significant source of microbiological contamination. For this reason, FaSSIF V1, V2, and V3 media were prepared using buffers that had been sterilized by autoclaving $(n=3)$. To further decrease the risk of contamination from other sources, media preparation was performed in a laminar flow bench (10). Media were stored at RT $\left(22^{\circ} \mathrm{C} \pm 1{ }^{\circ} \mathrm{C}\right)$ 
and $10-\mathrm{mL}$ samples were transferred into sterile tryptone soya broth at $24,48,72$, and $96 \mathrm{~h}$. In each case, the broth was visually observed for turbidity and precipitation over 14 days at RT, according to the specifications for sterility of the $9^{\text {th }}$ European Pharmacopeia (11). As a positive control, a saliva sample was added to the sterile tryptone soya and subjected to the same incubation conditions. It was not necessary to subject FeSSIF and FaSSGF to the microbiological test, as these media are produced from the same powder as FaSSIF V1.

\section{Cryogenic Transmission Electron Microscopy (Cryo- TEM)}

Cryo-TEM of FaSSIF V1, V2, and V3 was carried out to identify the colloidal structures present in the media. Sampling points are shown in Figure 1C. Vitrification of samples was performed with a plunge freezer, Vitrobot Mark IV (Thermo Fisher Scientific, Eindhoven, Netherlands). The relative humidity was set to $100 \%$, and blot time was adjusted to 4 seconds. A $4-\mu \mathrm{L}$ sample was transferred to a carbon-copper grid and frozen in liquid ethane at melting point. Grids were stored under liquid nitrogen until further analysis at $-196{ }^{\circ} \mathrm{C}$. A FEI Tecnai G2 F30 Twin TEM (Thermo Fisher Scientific) was used to evaluate the samples and a Falcon 3EC direct detector (Thermo Fisher Scientific) was used for image recording. The imaging process was performed under low-dose conditions.

\section{Statistics and Data Analysis}

Statistical evaluation was carried out using Microsoft Excel 2016 and SPSS Statistics (version 24, IBM). Data were reported as the arithmetic mean and standard deviation except for $\mathrm{pH}$ values, which were reported as the geometric mean together with the range.

A mixed model analysis of variance (ANOVA) was performed for the storage assessment at RT, assigning the measurement parameters ( $\mathrm{pH}$, surface tension, etc.) as 'within-subject' variables and time as the 'betweensubject' factor. The Type I error was set to 0.05 .

The paired t-test, unpaired t-test, Wilcoxon test, and Mann-Whitney U-test were used to evaluate results from the initial assessment and to compare results between the initial and extended storage assessments after an additional $24 \mathrm{~h}$ at $37^{\circ} \mathrm{C}$.

Where a significant difference was detected $(p \leq 0.05)$, the $p$-value is reported, whereas when the results were not statistically different $(p>0.05)$, no $p$-value is reported.

\section{RESULTS}

All results are available in full detail in the Supplementary Material available upon request from the authors.

\section{Physical Stability}

The results for the physical stability in terms of $\mathrm{pH}$, buffer capacity, surface tension, particle size, polydispersity index, and turbidity are summarized for all media in Figures 2 and 3.

\section{FaSSGF}

Room temperature storage: Values of $\mathrm{pH}$, surface tension, and turbidity did not change appreciably over the equilibration period of $2 \mathrm{~h}$. Moreover, the mean $\mathrm{pH}$ (1.58, range: $1.55-1.64)$, surface tension $(57.77 \pm 5.19$ $\mathrm{mN} / \mathrm{m})$, and turbidity ( $0.26 \pm 0.13 \mathrm{NTU})$ of FaSSGF also remained stable over the entire storage period of $96 \mathrm{~h}$ at RT. The buffer capacity could not be measured accurately for FaSSGF due to the lack of buffer components in this medium. Likewise, PCS measurements for particle size of FaSSGF were inconclusive due to the low concentration of particles.

Additional storage at $37^{\circ} \mathrm{C}$ : Samples of FaSSGF stored for $2 \mathrm{~h}$ at RT then exposed to $37{ }^{\circ} \mathrm{C}$ for 24 hours exhibited stable $\mathrm{pH}$ and turbidity. The surface tension decreased from $58.3 \pm 4.5 \mathrm{mN} / \mathrm{m}$ after $2 \mathrm{~h}$ at RT to $46.1 \pm 1.36 \mathrm{mN} / \mathrm{m}$ $(p=0.005)$ after the additional $24 \mathrm{~h}$ at $37^{\circ} \mathrm{C}$.

After $96 \mathrm{~h}$ of storage at RT, there was a slight increase in $\mathrm{pH}$ from $1.51(1.48-1.55)$ to $1.62(1.59-1.67)$ when the sample was additionally exposed to $37{ }^{\circ} \mathrm{C}$ for $24 \mathrm{~h}$. An increase in turbidity from 0.21 to 2.49 NTU $(p<0.001)$ was also recorded. The surface tension remained stable under these conditions.

The $\mathrm{pH}$ and surface tension were similar between the FaSSGF samples that had been stored at RT for $96 \mathrm{~h}$ then held at $37^{\circ} \mathrm{C}$ for $24 \mathrm{~h}$ and those stored for $2 \mathrm{~h}$ at RT, then held at $37^{\circ} \mathrm{C}$ for $24 \mathrm{~h}$. Although the turbidity increased to a higher value when exposed to $37^{\circ} \mathrm{C}$ for $24 \mathrm{~h}$ after $96 \mathrm{~h}$ at RT ( $2.49 \pm 0.47 \mathrm{NTU})$ compared to $2 \mathrm{~h}$ at RT $(0.18 \pm 0.07$ NTU, $p<0.001)$, the absolute value remained low.

\section{FaSSIF $\mathbf{1}$}

Room temperature storage: For FaSSIF V1, the mean $\mathrm{pH}$ $6.50,6.46-6.53)$, surface tension (51.65 $\pm 2.5 \mathrm{mN} / \mathrm{m})$, and buffer capacity $(9.49 \pm 0.3 \mathrm{mM} / \mathrm{l} / \Delta \mathrm{pH})$ were stable over the entire $96-\mathrm{h}$ storage period at RT. The turbidity of the media increased from the initial value $(6.79 \pm 6.34$ NTU) to $13.24 \pm 3.47 \mathrm{NTU}$ after $2 \mathrm{~h}$ of storage at RT, then increased further over the remainder of the 96 -h storage 

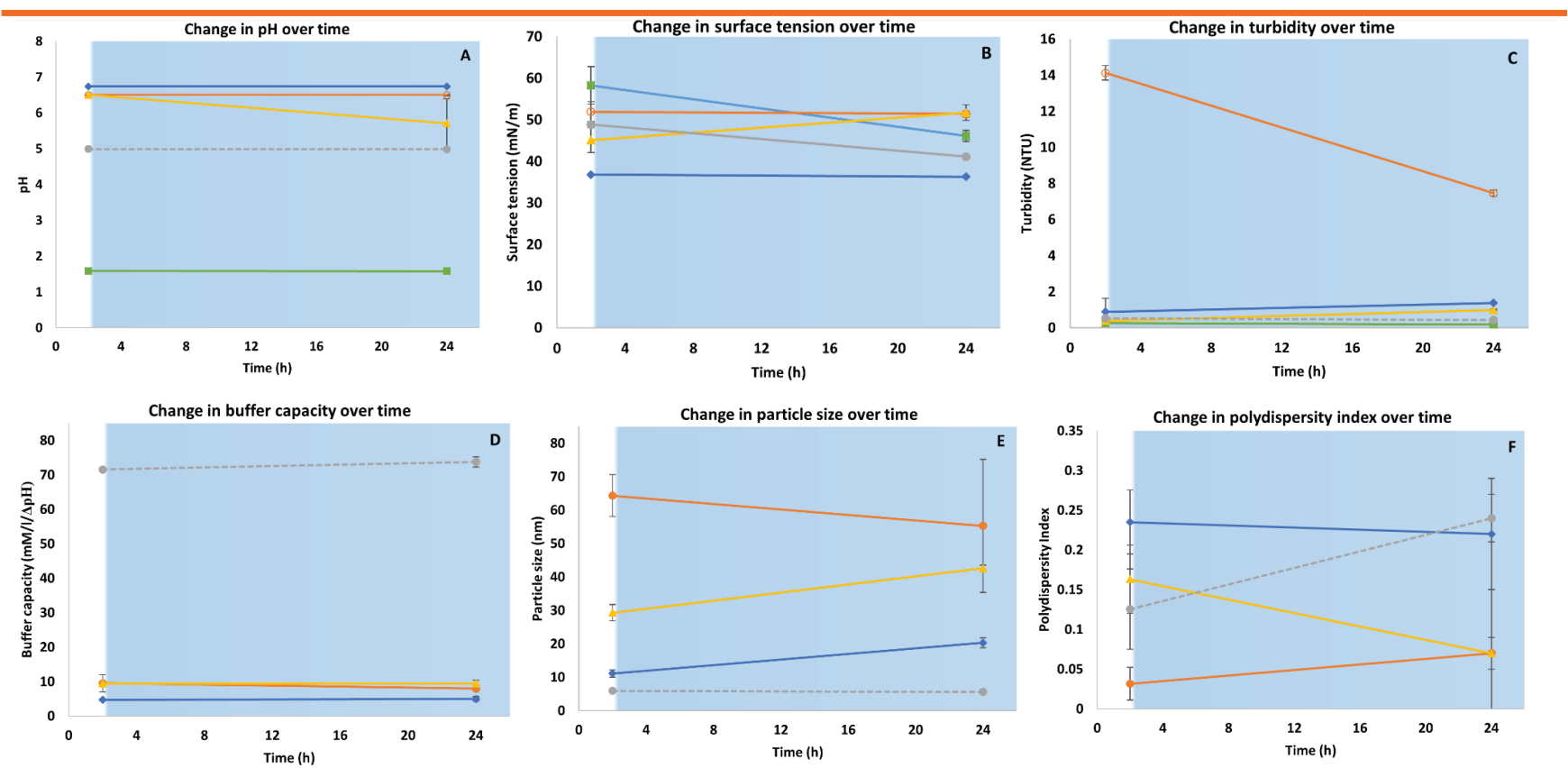

Figure 2. Results for the six parameters measured on various biorelevant media over a total of $24 \mathrm{~h}$ (includes $R T$ [white area] and $37^{\circ} \mathrm{C}$ [blue area]) for the initial assessment. In all panels, colored lines represent media as follows: green, FaSSGF; orange, FaSSIF V1; yellow, FaSSIF V2; blue, FaSSIF V3; gray, FeSSIF. FaSSGF, fasted-state gastric media; FaSSIF V1-V3, fasted-state intestinal media version 1-3; FeSSIF, fed-state intestinal media.

All results are available in tabular form in the supplemental material (available from authors).
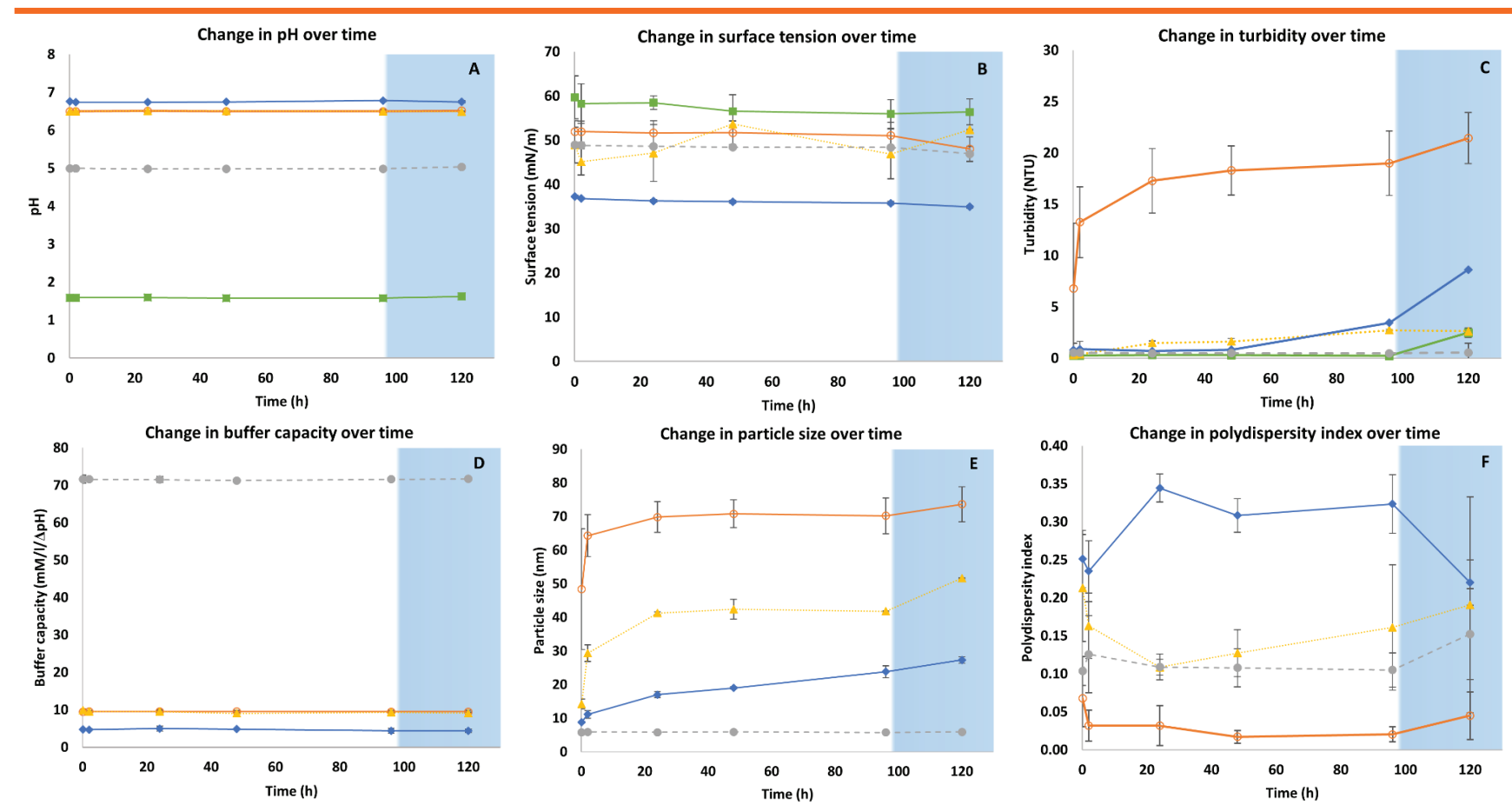

Figure 3. Results for the six parameters measured on various biorelevant media over a total of $120 \mathrm{~h}$ (includes $R T$ [white area] and $37^{\circ} \mathrm{C}$ [blue area]) for the assessment after extended storage. In all panels, colored lines represent media as follows: green, FaSSGF; orange, FaSSIF V1; yellow, FaSSIF V2; blue, FaSSIF V3; gray, FeSSIF. FaSSGF, fasted-state gastric media; FaSSIF V1-V3, fasted-state intestinal media version 1-3; FeSSIF, fed-state intestinal media.

All results are presented in tabular form in the supplementary material (available from authors). 
time to $18.99 \pm 3.15 \mathrm{NTU}$. The particle size increased from $48.38 \pm 17.93$ to $64.31 \pm 6.27 \mathrm{~nm}$ during the first $2 \mathrm{~h}$ at RT and then plateaued to a value of $70.21 \pm 5.31 \mathrm{~nm}$ after $96 \mathrm{~h}$ at RT. Although there is an apparent trend toward an increase in particle size with time for FaSSIF V1, values were not statistically significant different for any time point comparisons. The lack of significance in the results for particle size is attributable to high variability among individual measurements. The polydispersity index decreased from $0.07 \pm 0.04$ to $0.03 \pm 0.02$ during the first $2 \mathrm{~h}$ and remained extremely low $(0.033 \pm 0.028)$ over the rest of the storage time at RT.

Additional storage at $37{ }^{\circ} \mathrm{C}$ : None of the measured parameters varied between the values after $2 \mathrm{~h}$ of RT storage and after $24 \mathrm{~h}$ of storage at $37^{\circ} \mathrm{C}$.

Comparing the results from samples stored for $96 \mathrm{~h}$ at RT and after an additional $24 \mathrm{~h}$ at $37^{\circ} \mathrm{C}$, values of $\mathrm{pH}$, surface tension, buffer capacity, and polydispersity index remained stable. There was a minimal increase of turbidity under the same conditions from $18.99 \pm 3.15$ to $21.45 \pm$ 2.47 NTU $(p=0.049)$. Particle size also increased slightly to $73.62 \pm 5.19 \mathrm{~nm}$, but the increase was not significant.

Apart from turbidity (14.13 \pm 0.4 vs. $21.45 \pm 45, p=0.001)$, a comparison of parameters between samples exposed to $2 \mathrm{~h}$ at RT then held for $24 \mathrm{~h}$ at $37^{\circ} \mathrm{C}$ and those exposed to $96 \mathrm{~h}$ at RT then held for $24 \mathrm{~h}$ at $37^{\circ} \mathrm{C}$ did not reveal any significant differences.

\section{FaSSIF V2}

Room temperature storage: The mean $\mathrm{pH}$ (6.50, range, 6.49-6.52), surface tension ( $48.33 \pm 4.81 \mathrm{mN} / \mathrm{m})$, and buffer capacity $(9.43 \pm 0.46 \mathrm{mM} / \mathrm{l} / \Delta \mathrm{pH})$ for FaSSIF V2 were stable during the first $2 \mathrm{~h}$ and over the rest of the 96-h storage period at RT. A slight increase in turbidity was observed from $0.32 \pm 0.1$ to $0.39 \pm 0.1$ NTU during the first $2 \mathrm{~h}$, and the value increased further to $2.72 \pm 0.09$ NTU after $96 \mathrm{~h}(p<0.001)$. Particle size measurement revealed a gradual increase between the initial value and at $2 \mathrm{~h}$, from $14.19 \pm 1.46$ to $29.31 \pm 2.47 \mathrm{~nm}(p<0.001)$. By $24 \mathrm{~h}$, the particle size had reached $41.24 \pm 0.36 \mathrm{~nm}$. However, after $96 \mathrm{~h}$ at RT, there was no further increase, with a measured value of $41.79 \pm 0.16 \mathrm{~nm}$. The polydispersity index was $0.155 \pm 0.06$ and did not change significantly with time during storage at RT.

Additional storage at $37{ }^{\circ} \mathrm{C}$ : When the samples were equilibrated for $2 \mathrm{~h}$ then held at $37{ }^{\circ} \mathrm{C}$ for $24 \mathrm{~h}, \mathrm{pH}$, surface tension, buffer capacity and polydispersity index remained stable. The turbidity remained low, increasing only from $0.38 \pm 0.09$ to $0.99 \pm 0.5$ NTU ( $p=0.017)$, but the particle size increased from $29.31 \pm 2.47$ to $42.58 \pm$ $0.89 \mathrm{~nm}(p=0.016)$.

Other than the particle size, which increased from $41.79 \pm$ 0.16 to $51.67 \pm 0.12 \mathrm{~nm}(p<0.001)$, all other parameters remained stable when FaSSIF V2 was exposed to an additional $24 \mathrm{~h}$ at $37^{\circ} \mathrm{C}$ after being stored at RT for $96 \mathrm{~h}$.

Comparison of the data for samples subjected to $37{ }^{\circ} \mathrm{C}$ for $24 \mathrm{~h}$ after $2 \mathrm{~h}$ of storage at RT with those for samples stored at RT for $96 \mathrm{~h}$ then subjected to $37{ }^{\circ} \mathrm{C}$ for 24 $\mathrm{h}$ revealed no differences in $\mathrm{pH}$ and surface tension. However, there were statistically significant differences in values of turbidity $(0.99 \pm 0.5$ vs. $2.64 \pm 0.04 \mathrm{NTU}, p<$ $0.001)$, particle size ( $42.58 \pm 0.15$ vs. $51.67 \pm 0.12 \mathrm{~nm}, p$ $<0.001)$, and polydispersity index (0.07 \pm 0.01 vs. $0.19 \pm$ $0.01, p=0.001$ ).

\section{FaSSIF V3}

Room temperature storage: During the first $2 \mathrm{~h}$ of storage at $\mathrm{RT}$, mean $\mathrm{pH}(6.75$, range, 6.73-6.78), surface tension $(36.45 \pm 0.62 \mathrm{mN} / \mathrm{m})$, and buffer capacity $(4.72 \pm 0.45$ $\mathrm{mM} / \mathrm{l} / \Delta \mathrm{pH}$ ) values were stable and remained so over the rest of the 96-h storage period. The turbidity value remained low over the entire 96-h storage period at RT. In the first $48 \mathrm{~h}$, turbidity was stable at $0.81 \pm 0.64$ to $0.83 \pm 0.06 \mathrm{NTU}$, then increased gradually to $3.45 \pm 0.06$ NTU after $96 \mathrm{~h}(p<0.001)$. Results from dynamic light scattering revealed a slight particle size increase from $8.83 \pm 0.19$ to $11.13 \pm 1.12 \mathrm{~nm}$ during the first $2 \mathrm{~h}$ at RT. After that, there was a further increase in particle size during storage at RT to a value of $23.82 \pm 1.77 \mathrm{~nm}$ at the end of $96 \mathrm{~h}(p<0.001)$. The value of polydispersity index was stable over the first $2 \mathrm{~h}$ at RT $(0.25 \pm 0.04$ vs. $0.24 \pm$ 0.04 ) and averaged $0.292 \pm 0.053$ during the rest of the 96-h storage period at RT.

Additional storage at $37^{\circ} \mathrm{C}$ : Values of $\mathrm{pH}$, buffer capacity, and polydispersity index remained constant when exposed to $24 \mathrm{~h}$ at $37^{\circ} \mathrm{C}$ after $2 \mathrm{~h}$ at RT. By contrast, there were statistically significant changes in surface tension ( $p$ $=0.043)$, turbidity $(p=0.01)$ and particle size $(p=0.027)$. However, the absolute differences in surface tension (36.8 \pm 0.2 vs. $36.3 \pm 0.22 \mathrm{mN} / \mathrm{m})$, and turbidity $(0.88 \pm 0.74$ vs. $1.38 \pm 0.05 \mathrm{NTU}$ ) were very small. Similar to the results during storage at $\mathrm{RT}$, the particle size grew continuously during the $24 \mathrm{~h}$ at $37^{\circ} \mathrm{C}$ (from $11.1 \pm 1.1$ to $20.3 \pm 1.5 \mathrm{~nm}$ ).

When FaSSIF V 3 was exposed to $24 \mathrm{~h}$ at $37^{\circ} \mathrm{C}$ after storing at RT for $96 \mathrm{~h}$, no differences in $\mathrm{pH}$, surface tension, buffer capacity or particle size were observed. Turbidity increased slightly to $8.63 \pm 0.03$ NTU $(p=0.016)$ and 
polydispersity index from $0.1 \pm 0.02$ to $0.15 \pm 0.06$ ( $p=$ 0.002).

Results after incubation for $24 \mathrm{~h}$ at $37^{\circ} \mathrm{C}$ after $2 \mathrm{~h}$ at RT were also compared with those obtained after FaSSIF V3 was stored at RT for $96 \mathrm{~h}$ then exposed to $37^{\circ} \mathrm{C}$ for $24 \mathrm{~h}$. Values for $\mathrm{pH}$, surface tension, buffer capacity, and polydispersity index were similar, but turbidity (1.38 \pm 0.05 vs. $8.63 \pm 0.03$ NTU, $p<0.001)$ and particle size were higher $(20.3 \pm 1.53$ vs. $27.31 \pm 1.01 \mathrm{~nm}, p=0.05)$ when the RT storage time was longer.

\section{FeSSIF}

Room temperature storage: All measured values of FeSSIF for $\mathrm{pH}$ (mean 4.99, range 4.96-5.1), surface tension (48.63 $\pm 0.35 \mathrm{mN} / \mathrm{m})$, turbidity ( $0.5 \pm 0.1 \mathrm{NTU})$, buffer capacity $(71.51 \pm 0.63 \mathrm{mM} / \mathrm{l} / \Delta \mathrm{pH})$, particle size $(5.85 \pm 0.21 \mathrm{~nm})$, and polydispersity index $(0.11 \pm 0.029)$ were stable during storage at RT for the first $2 \mathrm{~h}$ and during the rest of the 96-h storage period.

Additional storage at $37{ }^{\circ} \mathrm{C}$ : When FeSSIF was exposed to $24 \mathrm{~h}$ at $37^{\circ} \mathrm{C}$ after being stored at RT for $2 \mathrm{~h}$, all parameters stayed within one standard deviation of the initial mean value except for the surface tension which decreased from $48.8 \pm 0.4$ to $41.14 \pm 0.12 \mathrm{mN} / \mathrm{m}(p<0.001)$.

Comparison after storage for $24 \mathrm{~h}$ at $37{ }^{\circ} \mathrm{C}$ following either 2 or $96 \mathrm{~h}$ at RT revealed very small but statistically significant differences in $\mathrm{pH}$ (mean 4.97 [range 4.93-5.00] vs. 5.03 [5.02-5.05], $p=0.01$ ), surface tension (41.14 \pm 0.12 vs. $46.92 \pm 1.69 \mathrm{mN} / \mathrm{m}, p=0.032$ ) and polydispersity index (0.24 \pm 0.03 vs. $0.14 \pm 0.01, p=0.02)$. Turbidity, buffer capacity, and particle size all remained stable.

\section{Microbiological Analysis}

Over the 14-day incubation period, no microbiological contamination was observed in any biorelevant media sample and there was no increase in turbidity in comparison with the negative control. By contrast, the positive control was turbid and small particles corresponding to microbial growth were observed within $48 \mathrm{~h}$.

\section{Cryogenic Transmission Electron Microscopy (Cryo- TEM)}

The results of Cryo-TEM for FaSSIF V1, V2, andV3 are shown in Figure 4.

\section{FaSSIF V1}

Various colloidal structures were observed in Cryo-TEM for FaSSIF V1. Each of the following structures were observed in at least one sample: vesicles, disc micelles, agglomerates of disc micelles, thread-like micelles, and globular micelles; however, only thread-like micelles could be consistently observed across all storage conditions and incubation times.

After $2 \mathrm{~h}$ at RT, both vesicles and thread-like structures were observed. The thread-like structures appear to be micellar in nature (Fig. 4, left panel, A-I and A-II) (12). When the equilibrated samples were incubated at $37^{\circ} \mathrm{C}$ for 24 hours, the thread-like micelles were still observed, but no vesicles were found. Instead, multi-layer structures with three or more layers appeared (Fig. 4, left, D-I and D-II). The layers may have consisted of stacks of individual units or may have been thread-like micelles that had folded back on themselves; the resolution was not sufficient to distinguish between these two possibilities. Gore et al. described similar structures as disc micelles, which are associated with each other by entropic forces (13). The presence of individual disc micelles in the sample (Fig. 4, left, D-I) support the possibility of disc aggregation.

After a total incubation time of $24 \mathrm{~h}$ at RT, globular micelles (which appear as small black dots in Fig. 4, left, B-I and B-II) were observed in addition to the vesicles and thread-like micelles. After $96 \mathrm{~h}$ of incubation at RT, vesicles and thread-like micelles were present (Fig. 4, left, C-I and C-II), but no globular micelles were identified. However, after subsequent incubation at $37{ }^{\circ} \mathrm{C}$ for $24 \mathrm{~h}$, the globular micelles were again observed on the carbon grid. In these samples, thread-like micelles but no vesicles were observed (Fig. 4, left, E-I and E-II).

\section{FaSSIF V2}

In FaSSIF V2 the following structures were observed in at least one sample: vesicles, disc micelles, thread-like micelles, and globular micelles, whereby the thread-like micelles were the most consistently observed structure.

Two hours after preparation, single globular micelles were observed in FaSSIF V2 (Fig. 4, middle panel, A-I and A-II). In addition to the globular micelles, vesicles and thread-like micelles were also observed on the grid after RT storage at 24 and $96 \mathrm{~h}$ (Fig. 4, middle, B-I, B-II, C-I, and $\mathrm{C}-\mathrm{II})$. At the end of incubation at $37^{\circ} \mathrm{C}$, either directly after the $2 \mathrm{~h}$ equilibration period or after $96 \mathrm{~h}$ of storage at RT, only thread-like micelles were observed (Fig. 4, middle, D-I, D-II, E-I, and E-II), and these appeared to be of various lengths.

\section{FaSSIF V3}

In FaSSIF V3, the following structures were observed in at least one sample: vesicles, disc micelles, and globular micelles. Disc micelles were the most consistently observed structure. 


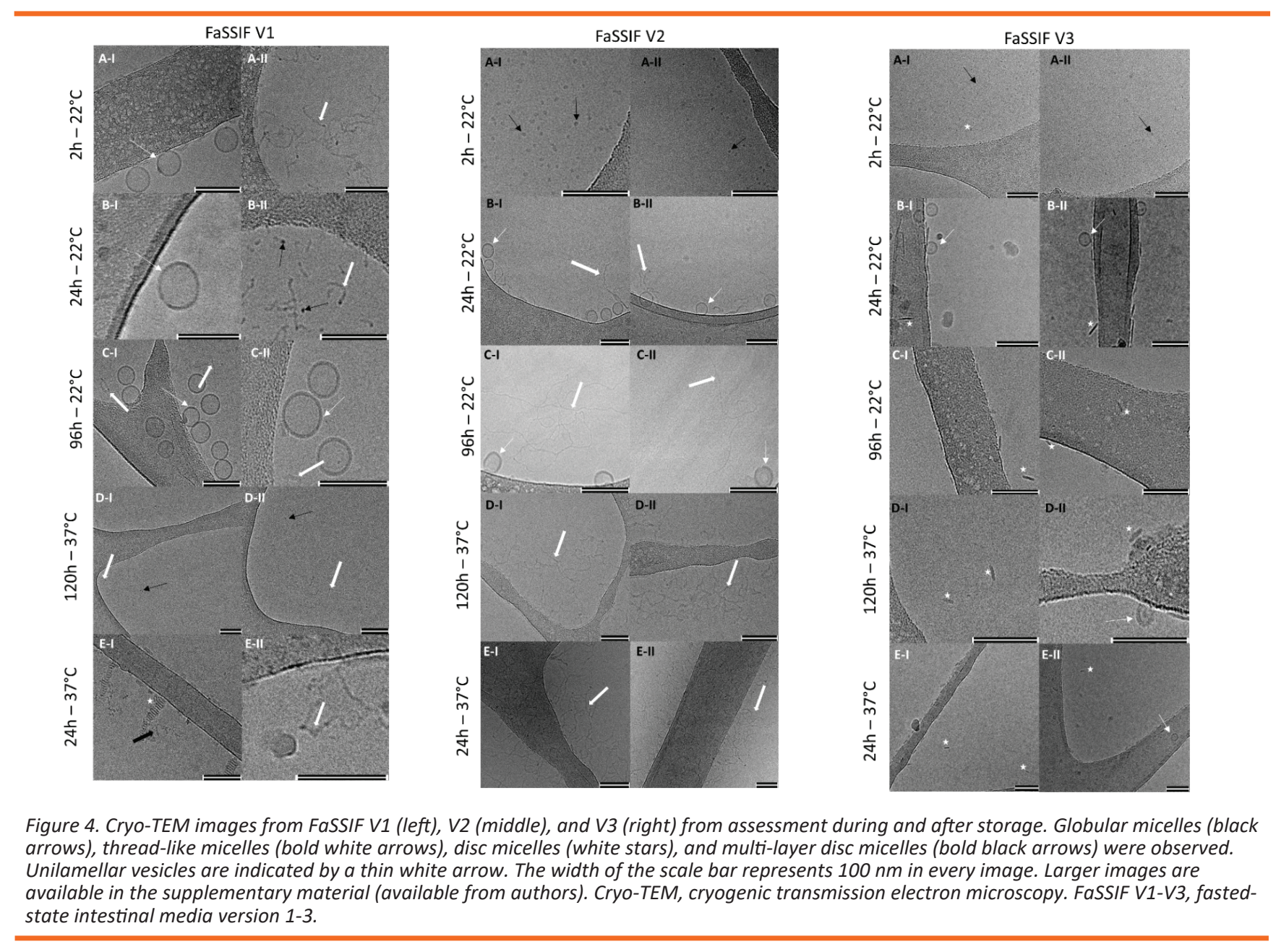

In FaSSIF V3, globular and disc micelles were present after the 2-h equilibration period at RT (Fig. 4, right panel, A-I and A-II). Disc micelles appeared to be stable in FaSSIF V3, as these structures were also observed after incubation at $37^{\circ} \mathrm{C}$ and after storage at RT for $96 \mathrm{~h}$, then after holding the sample for $24 \mathrm{~h}$ at $37^{\circ} \mathrm{C}$. Unilamellar vesicles were also observed in FaSSIF V3 in all samples except directly after reconstitution (Fig. 4, right, B-I, B-II, D-I, and D-II).

\section{DISCUSSION}

\section{Physical Stability}

\section{FaSSGF}

Measurements of particle size and Cryo-TEM were not conducted for FaSSGF because the concentration of phospholipids and bile salts in this medium are below the critical micellar concentration and thus the formation of stable colloidal structures is not expected $(14,15)$.

The only parameter which changed significantly during storage of FaSSGF was the turbidity. The European Union specifies values of turbidity for different classes of water. For example, tap water can have a turbidity value of up to 1.0 NTU (16). During storage of FaSSGF at RT, this limit was not exceeded. After storing FaSSGF for $96 \mathrm{~h}$ at RT then incubating at $37^{\circ} \mathrm{C}$ for $24 \mathrm{~h}$, the turbidity went up to $2.49 \pm 0.47$ NTU. Even so, the turbidity was minimal and not visually discernible.

Because all physicochemical parameters measured were essentially the same immediately after reconstitution and after the 2-h equilibration period, it can be concluded that this biorelevant medium can be used immediately after preparation from the instant powder. Based on the physicochemical parameters evaluated $(\mathrm{pH}$, surface tension, and turbidity), it was further concluded that FaSSGF is stable at RT for at least $96 \mathrm{~h}$ after preparation.

\section{FaSSIF V1}

After equilibration for $2 \mathrm{~h}$, the physicochemical parameters for FaSSIF V1 did not change significantly between 2 and $96 \mathrm{~h}$. These results are in accordance with previously published data and indicate that FaSSIF V1 is stable over a period of at least 4 days after reconstitution of the instant powder and equilibration 
and can be used for experiments within this timeframe (17).

However, several parameters changed significantly during the 2-h equilibration period at RT (turbidity, particle size, and polydispersity index). This appears to be associated with the changing nanostructures in FaSSIF V1 during the initial $2 \mathrm{~h}$ after reconstitution, leading to the conclusion that the equilibration period is necessary to achieve a reproducible particle size (18). In addition to the time factor for equilibration, Schurtenberger et al. indicated that changes in temperature of solutions containing bile salts and lecithin can alter the nanostructure and consequently, the particle size of the system (19). Thus, the equilibration of FaSSIF V1 should be carried out at RT.

Of the three versions of FaSSIF media, FaSSIF V1 has by far the greatest particle size and accordingly the highest turbidity. There is a non-linear relationship between the particle size and turbidity, hence a small increase in particle size leads to a nearly exponential increase in turbidity. In fact, the opalescence observed at the end of the 2-h equilibration period after preparation of FaSSIF V1 can be used as one of the criteria for quality control. A lack of opalescence may indicate incomplete formation of colloidal structures in the media, which would be reflected in particle size, particle size distribution, and turbidity measurements.

As turbidity increases there is a higher risk of interference due to background noise in, for example, ultraviolet (UV)-based analyses. However, with a robust analytical method such as high-performance liquid chromatography (HPLC)-UV, FaSSIF V1 can be used without reservation for solubility and dissolution experiments.

\section{FaSSIF V2}

Although the composition of FaSSIF V2 is similar to V1 (apart from the ratio of bile salt to lecithin), the time required to reach thermodynamic stability differed significantly between these media. Unlike the 2-h equilibration time required for V1, FaSSIF V2 needs up to $24 \mathrm{~h}$ to reach a stable particle size and turbidity. Potentially, the progressively increasing values of turbidity for FaSSIF V2 over the first $24 \mathrm{~h}$ at RT could pose a hurdle for reproducibility of dissolution results. This issue will need to be further investigated by comparing dissolution in FaSSIF V2 that is stored for $2 \mathrm{~h}$ at RT before use versus FaSSIF V2 stored for $24 \mathrm{~h}$ at RT before use. In the meantime, we recommend using a standard equilibration time for FaSSIF V2 across all experiments to ensure comparability of the results.
Between 24 and $96 \mathrm{~h}$ at RT, all physical parameters were stable and remained so when the sample was subjected to $24 \mathrm{~h}$ at $37^{\circ} \mathrm{C}$ after $96 \mathrm{~h}$ at RT, indicating that once the medium has equilibrated, the parameters are robust. These observations were supported by the similarity in Cryo-TEM structures in the samples.

\section{FaSSIF V3}

The third version of FaSSIF media differs significantly in the composition compared to the previous versions. The composition appears to have an influence on the evolution of the particle size, which continuously increased over time and did not reach a plateau within a week of storage at RT. Due to the complex composition of this medium, it is beyond the scope of this work to identify the specific components that lead to this behavior, although it has previously been reported that cholesterol can increase the equilibration time and decrease the particle size of biorelevant media $(18,20)$.

The $\mathrm{pH}$, surface tension, buffer capacity, and polydispersity index all remained stable over $96 \mathrm{~h}$ at RT, and turbidity values were consistently low.

As with FaSSIF V2, it remains to be seen whether the changing particle size in FaSSIF V3 has an influence on dissolution results. In the meantime, we recommend using a standard equilibration time for FaSSIF V3 across all experiments to ensure comparability of the results.

\section{FeSSIF}

FeSSIF displayed good stability with respect to all parameters at all temperature/time storage combinations tested. The high concentrations of bile salts and phospholipids present in this biorelevant medium enable rapid formation of a stable colloidal system. In contrast to FaSSIF versions, no equilibration time is required before use. FeSSIF can be used as soon as the powder is completely dissolved within the buffer system and a clear solution is observed. Based on the physicochemical analyses it can be concluded that FeSSIF is stable over a period of at least $96 \mathrm{~h}$ at RT and can be used for experiments within this time-frame.

\section{Particle Size Differences Among FaSSIF Versions}

Since the particle size varied among versions of FaSSIF, we examined potential causes of these differences. The main differences between the first two versions, FaSSIF V1 and V2, are the higher osmolality and a higher ratio of sodium taurocholate to lecithin in FaSSIF V1. Ionic strength, adjusted with sodium chloride in these media and reflected in the osmolality, influences the formation 
of colloidal structures. For example, the addition of sodium chloride to a system containing a constant ratio of lecithin and taurocholate is known to lead to a higher critical micelle concentration (CMC) and a smaller micellar size due to so-called "salting in" effects $(21,22)$. On this basis, it would be expected that FaSSIF V1 has a smaller particle size than V2.

In addition to osmolality, the ratio of bile salt to phospholipid can also affect the particle structure and size. In FaSSIF V1 this ratio is 4:1, but in FaSSIF V2 it is 15:1. Decreasing the ratio of bile salt to lecithin at a constant sodium chloride concentration decreases the CMC and leads to particle growth, as suggested by Shankland (23, 24). On this basis, it would be expected that $V 1$ has a larger particle size than V2. In FaSSIF V1, the effect of a smaller ratio of bile salts to phospholipids appears to override the effect of the higher osmolality effect, leading to a greater particle size in FaSSIF V1 than in FaSSIF V2.

The more complex composition of FaSSIF V3 makes a direct comparison with the two earlier versions difficult. The addition of cholesterol to a bile salt/lecithin system is known to decrease the micellar size to a much lower value (18). This is supported by the results from PCS and Cryo-TEM. Of course, the other constituents unique to FaSSIF V3 (glycocholate, lysolecithin, and sodium oleate) may also contribute to the differences in structures and particle size observed for FaSSIF V3.

\section{Microbiological Evaluation}

The absence of microbiological growth during incubation of biorelevant media in tryptone soya broth indicates that the instant powders are not a source of contamination. Microbial growth in the media during an experiment should therefore be interpreted as contamination by either the equipment or the buffer solution used to reconstitute the medium. To decrease the risk of microbiological growth during experiments of long duration ( $24 \mathrm{~h}$ or more), it is recommended that the buffer system be prepared with water of appropriate microbiological quality.

\section{Structures Observed in Cryo-TEM}

Various papers have analyzed biorelevant media with the Cryo-TEM technique. Similar to our findings, Kloefer et al. observed globular micelles, disc micelles, and vesicles in FaSSIF V1 (17). On the other hand, Riethorst et al. identified micelles of various sizes $(20-40 \mathrm{~nm})$ in FaSSIF V1, which appeared to mostly consist of globular micelles (25). It is possible that no vesicles were detected in the Riethorst study due to differences in the microscopy set-up and sample treatment (e.g., whether or not the samples were centrifuged). The age of the sample at the time of the vitrification process should also be considered, as the medium can change over time depending on the composition.

To enable a better understanding of biorelevant media at a colloidal level, we examined the time-dependency of the structures during storage at RT. To ensure that the nanostructures identified in the Cryo-TEM would be identical to those in the original samples, they were not centrifuged prior to vitrification.

As expected, the differences in buffer system, $\mathrm{pH}$, osmolality, and composition of the phospholipids and bile salts in the various versions of FaSSIF affect the type of colloidal structures present in the media. These differences are reflected in the particle size as well. The observed globular micelles had a particle size in the range between 5 and $20 \mathrm{~nm}$ in most cases, whereas disc micelles were slightly larger, at around 20 to $40 \mathrm{~nm}$. Stacks of disc micelles can reach sizes of up to $100 \mathrm{~nm}$ and greater, depending on how many individual disc micelles are involved. Particle size measurement of thread-like micelles is difficult due to the high convolution of the particles. Further, they appear to be of varying length and thus no reliable estimation of the particle size is possible. The size of vesicles is usually between 30 and $100 \mathrm{~nm}$. The structures observed by Cryo-TEM were generally in agreement with the PCS data during the assessment at RT, especially for those media containing vesicles and disc-like micelles.

The different sizes and types of micelles and vesicles that have been identified in human intestinal fluid (HIF) were also observed in one or more of the biorelevant media, including micelles within the range 10 to $50 \mathrm{~nm}$, threadlike micelles, and unilamellar and multilamellar vesicles (25-27). Comparison of structures that are present in fasted-state HIF with the biorelevant media does not explicitly favor one version of FaSSIF over the others, but rather, they complement one another in terms of representing the structures identified in the luminal aspirates.

\section{Potential Ramifications of Colloidal Structure for Solubility}

The solubility of nonpolar compounds is influenced, among other factors, by micellar and vesicular structures. Micelles with an asymmetric shape, such as thread-like micelles, have a larger core volume than round micelles, leading to the assumption that they will have a larger effect 
on improving solubility (28). Such thread-like micelles are present in FaSSIF V1 and V2 stored at $37^{\circ} \mathrm{C}$. Due to the larger particle size of FaSSIF V1, the total nonpolar area must be greater than V2, enabling an increased solubility of nonpolar compounds (under the assumption that the number of micelles stays the same). In FaSSIF V3 smaller micelles are found, but there are also vesicles present, and moreover, the presence of cholesterol will have an effect on the hydrophobic structures (18). Solubility experiments are therefore necessary to determine which of the versions best represents the conditions in vivo. Söderlind et al. determined that neutral compounds had a higher solubility in FaSSIF V1 than V2 (29). This is also in accordance with the data by Fuchs et al. (7). Moreover, both ionized and neutral compounds were observed to have an equal or higher solubility in FaSSIF V3 than V2 (7).

\section{Potential Ramification of Colloidal Structures for Stability}

Cryo-TEM analyses generally support the assumptions from the physical stability test regarding media stability over time. The constant values of turbidity, particle size, and polydispersity index of FaSSIF V1 are reflected by a stable particle structure in all samples stored at RT. The behavior of FaSSIF V2 was analogous here too, the observed structures were more similar once the medium had been equilibrated (i.e., after $24 \mathrm{~h}$ ). The influence of storage at a higher temperature $\left(37^{\circ} \mathrm{C}\right)$ not only increased the general particle size but also changed the types of particles observed in Cryo-TEM in both FaSSIF V1 and V2. By contrast, the colloidal structures observed in FaSSIF V3 could not be completely linked with the PCS results. Based on these observations, it seems that although CryoTEM is useful to identify trends, there is no consistent correlation with particle size.

\section{CONCLUSIONS}

Various biorelevant media are commercially available as powders for reconstitution. Due to the differences in composition, biorelevant media exhibit different colloidal structures and physicochemical properties as well as evolution with time and temperature. The studies presented here enable several conclusions to be made about implementation of biorelevant media in the laboratory.

FaSSGF, representing fasted-state conditions in the stomach, can be used immediately after constitution, no equilibration time is required. It can be stored at RT for at least $96 \mathrm{~h}$ before use without any significant alterations in its physicochemical properties.
For FaSSIF versions, representing fasted-state intestinal conditions, a standard equilibration period for the medium is necessary. FaSSIF V1 requires an equilibration time of $2 \mathrm{~h}$, and FaSSIF V2 requires $24 \mathrm{~h}$ to reach stable colloidal structures and physicochemical parameters. The parameters of FaSSIF V3 evolve over time. For both FaSSIF V2 and V3, it is recommended that, until the ramifications of evolving parameters with respect to solubility determination and dissolution testing have been evaluated, a standard waiting period (e.g., 2 h) between reconstitution and application is invoked across all experiments to ensure comparability of results.

Like FaSSGF, FeSSIF, which represents fed-state intestinal conditions, can be used immediately after reconstitution, as it equilibrates rapidly. Since its physicochemical parameters remain stable for at least $96 \mathrm{~h}$ at RT, it can be stored for several days at RT before use.

Microbiological contamination of the biorelevant medium by the instant powder is not an issue. However, the user is advised to use water and buffers of adequate microbial quality. For experiments of long duration (> $24 \mathrm{~h}$ ), it is recommended to autoclave the buffer solution.

\section{SUPPLEMENTAL MATERIAL}

The supplemental material is available from the author upon request.

\section{CONFLICT OF INTEREST}

The authors disclosed no potential conflicts of interest related to this article.

\section{REFERENCES}

1. Galia, E.; Nicolaides, E.; Horter, D.; Lobenberg, R.; Reppas, C.; Dressman, J. B. Evaluation of various dissolution media for predicting in vivo performance of class I and II drugs. Pharmaceutical research 1998, 15, 698-705. DOI: 10.1023/A:1011910801212.

2. Fiolka, T.; Dressman, J. Development, current applications and future roles of biorelevant two-stage in vitro testing in drug development. J. Pharm. Pharmacol. 2018, 70, 335-348. DOI: 10.1111/jphp.12875.

3. Morgan, S.; Grootendorst, P.; Lexchin, J.; Cunningham, C.; Greyson, D. The cost of drug development: A systematic review. Health Policy. 2011, 100, 4-17. DOI: 10.1016/j. healthpol.2010.12.002.

4. Otsuka, K.; Shono, Y.; Dressman, J. Coupling biorelevant dissolution methods with physiologically based pharmacokinetic modelling to forecast in-vivo performance of solid oral dosage 
forms. J. Pharm. Pharmacol. 2013, 65, 937-952. DOI: 10.1111/ jphp.12059.

5. Mann, J.; Dressman, J.; Rosenblatt, K.; Ashworth, L.; Muenster, U.; Frank, K.; Hutchins, P.; Williams, J.; Klumpp, L.; Wielockx, K.; Berben, P.; Augustijns, P.; Holm, R.; Hofmann, M.; Patel, S.; Beato, S.; Ojala, K.; Tomaszewska, I.; Bruel, J.-L.; Butler, J. Validation of dissolution testing with biorelevant media: An OrBiTo study. Mol. Pharm. 2017, 14, 4192-4201. DOI: 10.1021/ acs.molpharmaceut.7b00198.

6. Biorelevant.com. Preparation Calculators. https://biorelevant. com/fassif-fessif-fassgf/buy/. Accessed April 1, 2019.

7. Fuchs, A. FaSSIF-V3: [Development of a new generation of biorelevant media to simulate conditions in the human fasted small intestine.] Dissertation [Online], Goethe Universität Frankfurt am Main, Frankfurt am Main, 2015. https://dnb.info/1101377232/04. Accessed March 29, 2019. DOI: 10.1163/1872-9037_afco_asc_1232.

8. Litou, C.; Vertzoni, M.; Goumas, C.; Vasdekis, V.; Xu, W.; Kesisoglou, F.; Reppas, C. Characteristics of the human upper gastrointestinal contents in the fasted state under hypo- and A-chlorhydric gastric conditions under conditions of typical drug - drug interaction studies. Pharm. Res. 2016, 33, 1399-1412. DOI: 10.1007/s11095-016-1882-8.

9. Bergström, C. A. S.; Holm, R.; Jørgensen, S. A.; Andersson, S. B. E.; Artursson, P.; Beato, S.; Borde, A.; Box, K.; Brewster, M.; Dressman, J.; Feng, K.-I.; Halbert, G.; Kostewicz, E.; McAllister, M.; Muenster, U.; Thinnes, J.; Taylor, R.; Mullertz, A. Early pharmaceutical profiling to predict oral drug absorption: Current status and unmet needs. Eur. J. Pharm. Sci. 2014, 57, 173-199. DOI: 10.1016/j.ejps.2013.10.015.

10. Sterile Drug Products Produced by Aseptic Processing - Current Good Manufacturing Practice; Gudeance for Industry; U.S. Department of Health and Human Services, Food and Drug Administration, Center for Drug Evaluation and Research (CDER), Center for Biologics Evaluation and Research (CBER), Office of Regulatory Affairs (ORA).U.S. Government Printing Office, Washington, DC, 2004.

11. 9.0/2.6.1 Sterility Test, European Pharmacopoeia, 9th ed. German Pharmacists' Publisher, Eschborn, 2017. DOI: 10.1002/ ardp.19753081016.

12. Cohen, D. E.; Thurston, G. M.; Chamberlin, R. A.; Benedek, G. B.; Carey, M. C. Laser light scattering evidence for a common wormlike growth structure of mixed micelles in bile salt- and straight-chain detergent-phosphatidylcholine aqueous systems: Relevance to the micellar structure of bile. Biochemistry 1998, 37, 14798-14814. DOI: 10.1021/bi980182y.

13. Gore, T.; Dori, Y.; Talmon, Y.; Tirrell, M.; Bianco-Peled, H. Selfassembly of model collagen peptide amphiphiles. Langmuir 2001, 17, 5352-5360. DOI: 10.1021/la010223i.

14. Carey, M. C.; Small, D. M. The characteristics of mixed micellar solutions with particular reference to bile. Am. J. Med. 1970, 49, 590-608. DOI: 10.1016/S0002-9343(70)80127-9.

15. Yu. A. Shchipunov; Somasundaran, P.; Hubbard, A. T. Lecithin // Encyclopedia of Surface and Colloid Science; Marcel Dekker: New York, 2002.

16. Proposal for a Directive of the European Parliament and of the Council on the quality of water intended for human consumption (recast). European Commission, January 2, 2018.

17. Kloefer, B.; van Hoogevest, P.; Moloney, R.; Kuentz, M.; Leigh, M. L.S.; Dressman, J. Study of a standardized taurocholate- lecithin powder for preparing the biorelevant media FeSSIF and FaSSIF. Dissolut. Technol. 2010, 17, 6-13. DOI: 10.14227/DT170310P6.

18. Khoshakhlagh, P.; Johnson, R.; Langguth, P.; Nawroth, T.; Schmueser, L.; Hellmann, N.; Decker, H.; Szekely, N. K. Fasted-state simulated intestinal fluid "FaSSIF-C", a cholesterol containing intestinal model medium for in vitro drug delivery development. J Pharm. Sci. 2015, 104, 2213-2224. DOI: 10.1002/jps.24470.

19. Schurtenberger, P.; Mazer, N.; Kaenzig, W. Micelle to vesicle transition in aqueous solutions of bile salt and lecithin. J. Phys. Chem. 1985, 89, 1042-1049. DOI: 10.1021/j100252a031.

20. Almgren, M.; Edwards, K.; Karlsson, G. Cryo transmission electron microscopy of liposomes and related structures. Colloids Surf. A Physicochem. Eng. Asp. 2000, 174, 3-21. DOI: 10.1016/S09277757(00)00516-1.

21. Nair, P. P.; Kritchevsky, D. The Bile Acids Chemistry, Physiology, and Metabolism: Volume 1: Chemistry; Springer US; Imprint; Springer: Boston, MA, 1971.

22. Stuart, M. C. A.; Boekema, E. J. Two distinct mechanisms of vesicle-to-micelle and micelle-to-vesicle transition are mediated by the packing parameter of phospholipid-detergent systems. Biochim. Biophys. Acta. 2007, 1768, 2681-2689. DOI: 10.1016/j. bbamem.2007.06.024.

23. Shankland, $\mathbf{W}$. The equilibrium and structure of lecithin-cholate mixed micelles. Chem. Phys. Lipids. 1970, 4, 109-130. DOI: 10.1016/0009-3084(70)90042-3.

24. Leng, J.; Egelhaaf, S. U.; Cates, M. E. Kinetics of the micelle-tovesicle transition: aqueous lecithin-bile salt mixtures. Biophys. J. 2003, 85, P1624-1646. DOI: 10.1016/S0006-3495(03)74593-7.

25. Riethorst, D.; Baatsen; Remijn, C.; Mitra, A.; Tack, J.; Brouwers, J.; Augustijns, P. An in-depth view into human intestinal fluid colloids: intersubject variability in relation to composition. Mol. Pharm. 2016, 13, 3484-3493. DOI: 10.1021/acs. molpharmaceut.6b00496.

26. Müllertz, A.; Reppas, C.; Psachoulias, D.; Vertzoni, M.; Fatouros, D. G. Structural features of colloidal species in the human fasted upper small intestine. J. Pharm. Pharmacol. 2015, 67, 486-492. DOI: 10.1111/jphp.12336.

27. Müllertz, A.; Fatouros, D. G.; Smith, J. R.; Vertzoni, M.; Reppas, C. Insights into intermediate phases of human intestinal fluids visualized by atomic force microscopy and cryo-transmission 
electron microscopy ex vivo. Mol. Pharm. 2012, 9, 237-247. DOI: 10.1021/mp200286x.

28. Rangel-Yagui, C. O.; Pessoa, A.; Tavares, L. C. Micellar solubilization of drugs. J. Pharm. Sci. 2005, 8, 147-165.
29. Söderlind, E.; Karlsson, E.; Carlsson, A.; Kong, R.; Lenz, A.; Lindborg, S.; Sheng, J. J. Simulating fasted human intestinal fluids: Understanding the roles of lecithin and bile acids. Mol. Pharm. 2010, 7, 1498-1507. DOI: 10.1021/mp100144v. 